\title{
Improving KKM Theory on General Metric Type Spaces
}

https://doi.org/10.1515/taa-2020-0101

Received March 9, 2020; accepted May 10, 2021

\begin{abstract}
A generalized metric type space is a generic name for various spaces similar to hyperconvex metric spaces or extensions of them. The purpose of this article is to introduce some KKM theoretic works on generalized metric type spaces and to show that they can be improved according to our abstract convex space theory. Most of these works are chosen on the basis that they can be improved by following our theory. Actually, we introduce abstracts of each work or some contents, and add some comments showing how to improve them.
\end{abstract}

Keywords: Abstract convex space, KKM theorem, KKM space, map classes $\mathfrak{K C}, \mathfrak{K O}$

MSC: 46A03, 47H10, 49J53, 54C60, 54H25, 91A11, 91B02

\section{Introduction}

The KKM theory, first called by the author in 1992 [17], is the study on applications of equivalent formulations or generalizations of the KKM theorem due to Knaster, Kuratowski, and Mazurkiewicz in 1929. The KKM theorem is one of the most well-known and important existence principles and provides the foundations for many of the modern essential results in diverse areas of mathematical sciences. Since the theorem and its many equivalent formulations or extensions are powerful tools in showing the existence of solutions of a lot of problems in pure and applied mathematics, many scholars have been studying its further extensions and applications.

The KKM theory was first devoted to convex subsets of topological vector spaces mainly by Ky Fan and Granas, and later to the so-called convex spaces by Lassonde, to $c$-spaces by Horvath and others, to G-convex spaces mainly by the present author. In 2006-09, we proposed new concepts of abstract convex spaces and partial KKM spaces which are proper generalizations of G-convex spaces and adequate to establish the KKM theory. Now the KKM theory becomes the study of abstract convex spaces due to ourselves in 2006 [28] and we obtained a large number of new results in such frame. For the history of the KKM theory, see our recent article [29].

Almost independently to the above progress, Khamsi in 1996 [11] found that hyperconvex metric spaces are partial KKM spaces, and obtained some KKM theoretic results. Motivated by his work, in the last decade, many authors obtained some KKM theoretic results on hyperconvex metric spaces as well as newly defined spaces like $\mathcal{N} \mathcal{R}$-metric spaces, metric type spaces, cone metric spaces, cone b-metric spaces, tvs-cone metric spaces, circular metric spaces, modular metric spaces, convex metric spaces, etc. These type of spaces may be called generalized metric type spaces as a generic name.

The purpose of this article is to introduce some KKM theoretic works on generalized metric type spaces and to show that they can be improved according to our abstract convex space theory.

\footnotetext{
^Corresponding Author: Sehie Park: The National Academy of Sciences, Republic of Korea, Seoul 06579, Korea; and Department of Mathematical Sciences, Seoul National University, Seoul 08826, Korea, E-mail: park35@snu.ac.kr; sehiepark@gmail.com; http://parksehie.com
} 
In Section 2, we introduce some basic things on our abstract convex spaces as a preliminary. Section 3 deals with various properties of (partial) KKM spaces that can be applied various particular types of spaces.

In Section 4, we recall previous studies on generalized metric type spaces and basic facts on them. Section 5 deals with several works on the generalized metric type spaces. Most of these works are chosen on the basis that they can be improved by following our KKM theory on abstract convex spaces. Actually, we introduce abstracts of each work or some contents of them, and add some comments showing how to improve them. Finally in Section 6 with the conclusion, we propose some suggestions to improve the study of general metric type Spaces.

This paper is parallel to our previous work [32] in 2020 in the sense that we repeat some introductory part there for reader's convenience. Note that there are scores of new metric type spaces appeared in the metric fixed point theory related to contractive type maps or nonexpansive maps. But we will not concerned with them in this paper.

\section{Abstract convex spaces}

In order to upgrade the KKM theory, in 2006-12, we proposed new concepts of abstract convex spaces and the (partial) KKM spaces which are proper generalizations of various known types of particular spaces and adequate to establish the KKM theory.

Multimaps are also called simply maps. Let $\langle D\rangle$ denote the set of all nonempty finite subsets of a set $D$.

Recall the following in [23]:

Definition. An abstract convex space $(E, D ; \Gamma)$ consists of a topological space $E$, a nonempty set $D$, and a multimap $\Gamma:\langle D\rangle \multimap E$ with nonempty values $\Gamma_{A}:=\Gamma(A)$ for $A \in\langle D\rangle$, such that the $\Gamma$-convex hull of any $D^{\prime} \subset D$ is denoted and defined by

$$
\operatorname{co}_{\Gamma} D^{\prime}:=\bigcup\left\{\Gamma_{A} \mid A \in\left\langle D^{\prime}\right\rangle\right\} \subset E .
$$

A subset $X$ of $E$ is called a $\Gamma$-convex subset of $(E, D ; \Gamma)$ relative to $D^{\prime}$ if for any $N \in\left\langle D^{\prime}\right\rangle$, we have $\Gamma_{N} \subset X$, i.e., $\operatorname{co}_{\Gamma} D^{\prime} \subset X$. Let $(E ; \Gamma):=(E, E ; \Gamma)$.

Definition. Let $(E, D ; \Gamma)$ be an abstract convex space and $Z$ a topological space. For a multimap $F: E \multimap Z$ with nonempty values, if a multimap $G: D \multimap Z$ satisfies

$$
F\left(\Gamma_{A}\right) \subset G(A):=\bigcup_{y \in A} G(y) \quad \text { for all } A \in\langle D\rangle,
$$

then $G$ is called a KKM map with respect to $F$. A KKM map $G: D \multimap E$ is a KKM map with respect to the identity map $1_{E}$ of $E$.

A multimap $F: E \multimap Z$ is called a $\mathfrak{K C}$-map [resp. a $\mathfrak{K O}-m a p$ ] if, for any closed-valued [resp. open-valued] KKM map $G: D \multimap Z$ with respect to $F$, the family $\{G(y)\}_{y \in D}$ has the finite intersection property. In this case, we denote $F \in \mathfrak{K C}(E, Z)$ [resp. $F \in \mathfrak{K O}(E, Z)]$.

Remark. Motivated our old works, Chang and Yen [5] defined a generalized KKM map with respect to $F$ instead of our KKM map with respect to $F$, and called the KKM family for our $\mathfrak{K C}$. Many authors used such obsolete terminology of Chang and Yen,

Example. A typical example of the abstract convex spaces is the following type of spaces:

Definition. A $\phi_{A}$-space $\left(X, D ;\left\{\phi_{A}\right\}_{A \in\langle D\rangle}\right)$ consists of a topological space $X$, a nonempty set $D$, and a family of continuous functions $\phi_{A}: \Delta_{n} \rightarrow X$ (that is, singular $n$-simplices) for $A \in\langle D\rangle$ with $|A|=n+1$. By putting $\Gamma_{A}:=\phi_{A}\left(\Delta_{n}\right)$ for each $A \in\langle D\rangle$, the triple $(X, D ; \Gamma)$ becomes an abstract convex space.

Definition. For a $\phi_{A}$-space $\left(X, D ;\left\{\phi_{A}\right\}\right)$, any multimap $G: D \multimap X$ satisfying

$$
\phi_{A}\left(\Delta_{J}\right) \subset G(J) \text { for each } A \in\langle D\rangle \text { and } J \in\langle A\rangle
$$


is called a KKM map.

Lemma. Let $(X, D ; \Gamma)$ be a $\phi_{A}$-space and $G: D \multimap X$ a multimap with nonempty closed [resp. open] values. Suppose that $G$ is a KKM map. Then $\{G(a)\}_{a \in D}$ has the finite intersection property.

Now we have the following diagram for subclasses of abstract convex spaces $(E, D ; \Gamma)$ :

$$
\begin{gathered}
\text { Simplex } \Rightarrow \text { Convex subset of a t.v.s. } \Rightarrow \text { Lassonde’s convex space } \\
\qquad \text { Horvath space } \Rightarrow \text { G-convex space } \Leftrightarrow \phi_{A} \text {-space } \\
\Rightarrow \text { KKM space } \Rightarrow \text { Partial KKM space } \\
\Rightarrow \text { Abstract convex space. }
\end{gathered}
$$

In order to introduce a most general form of the KKM type theorem, consider the following related four conditions for a map $G: D \multimap Z$ with a topological space $Z$ :
(a) $\bigcap_{y \in D} \overline{G(y)} \neq \emptyset$ implies $\bigcap_{y \in D} G(y) \neq \emptyset$.
(b) $\bigcap_{y \in D} \overline{G(y)}=\overline{\bigcap_{y \in D} G(y)}(G$ is intersectionally closed-valued).
(c) $\bigcap_{y \in D} \overline{G(y)}=\bigcap_{y \in D} G(y)$ ( $G$ is transfer closed-valued $)$.
(d) $G$ is closed-valued.

Recall the following one of the most general KKM type theorems in [25] and others:

Theorem C. Let $(E, D ; \Gamma)$ be an abstract convex space, $Z$ a topological space, $F \in \mathfrak{K C}(E, D, Z)$, and $G: D \multimap Z$ a map such that

(1) $\bar{G}$ is a KKM map w.r.t. F; and

(2) there exists a nonempty compact subset $K$ of $Z$ such that either

(i) $K=Z$;

(ii) $\bigcap\{\overline{G(y)} \mid y \in M\} \subset K$ for some $M \in\langle D\rangle$; or

(iii) for each $N \in\langle D\rangle$, there exists a $\Gamma$-convex subset $L_{N}$ of $E$ relative to some $D^{\prime} \subset D$ such that $N \subset D^{\prime}$, $\overline{F\left(L_{N}\right)}$ is compact, and

$$
\overline{F\left(L_{N}\right)} \cap \bigcap_{y \in D^{\prime}} \overline{G(y)} \subset K .
$$

Then we have

$$
\overline{F(E)} \cap K \cap \bigcap_{y \in D} \overline{G(y)} \neq \emptyset
$$

Furthermore,

$(\alpha)$ if $G$ is transfer closed-valued, then $\overline{F(E)} \cap K \cap \bigcap\{G(y) \mid y \in D\} \neq \emptyset$; and

$(\beta)$ if $G$ is intersectionally closed-valued, then $\bigcap\{G(y) \mid y \in D\} \neq \emptyset$.

The following fact was given in [21].

Theorem D. Let $(E, D ; \Gamma)$ be an abstract convex space, $Z, W$ two topological spaces, $F \in \mathfrak{K C}(E, Z)$ and $f: Z \rightarrow$ $W$ a continuous function. Then $f F \in \mathfrak{K C}(E, W)$. This also holds for $\mathfrak{K O}$ instead of $\mathfrak{K C}$.

The reader will find a large number of examples of this Theorem in the papers listed in Section 5.

\section{The KKM theory on abstract convex spaces}

In the first half of this section, we introduce some typical KKM theoretic results on abstract convex spaces appeared mainly in $[23,24,29]$. Of course we can not repeat whole works on that subject. So in the second half of this section, we state some subjects of our study on the KKM theory without mentioning the literature. 
An abstract convex space $(E, D ; \Gamma)$ is called a KKM space whenever the following holds:

(0) The KKM principle. For any closed-valued [resp. open-valued] KKM map $G: D \multimap E$, the family $\{G(z)\}_{z \in D}$ has the finite intersection property.

This is equivalent to each one of the following and others:

The Fan matching property

Another finite intersection property

The geometric property or the section property

The Fan-Browder fixed point property

Existence of maximal elements

Analytic formulation

Minimax inequality

Analytic alternative

An abstract convex space $(E, D ; \Gamma)$ is called a partial KKM space whenever the following partial condition of (0) holds:

(0)' The partial KKM principle. For any closed-valued KKM map $G: D \multimap E$, the family $\{G(y)\}_{y \in D}$ has the finite intersection property.

This is equivalent to each partial one (that is, closed-valued case) of the above list.

Theorem. For a compact partial KKM space $(X ; \Gamma)$, the following statements hold.

Several types of minimax inequalities

Several types of variational inequalities

Let $\left(X ; \Gamma_{1}\right)$ and $\left(Y ; \Gamma_{2}\right)$ be abstract convex spaces. For their product, we can define $\Gamma_{X \times Y}(A):=\Gamma_{1}\left(\pi_{1}(A)\right) \times$ $\Gamma_{2}\left(\pi_{2}(A)\right)$ for $A \in\langle X \times Y\rangle$, where $\pi_{1}: X \times Y \rightarrow X$ and $\pi_{2}: X \times Y \rightarrow Y$ are projections.

Theorem. For a compact product partial KKM space $(E ; \Gamma):=\left(X \times Y ; \Gamma_{X \times Y}\right)$, the following statements hold.

The basic minimax theorem

Generalized von Neumann-Sion minimax theorem

Collective fixed point theorem

The von Neumann-Fan intersection theorem

The Fan type analytic alternative

Generalized Nash-Fan type equilibrium theorem

Since we first named the KKM theory in 1992 [17], we have tried to improve the theory by applying such basic theory of abstract convex spaces. In fact, we have studied the KKM theory with respect to the following list of subjects:

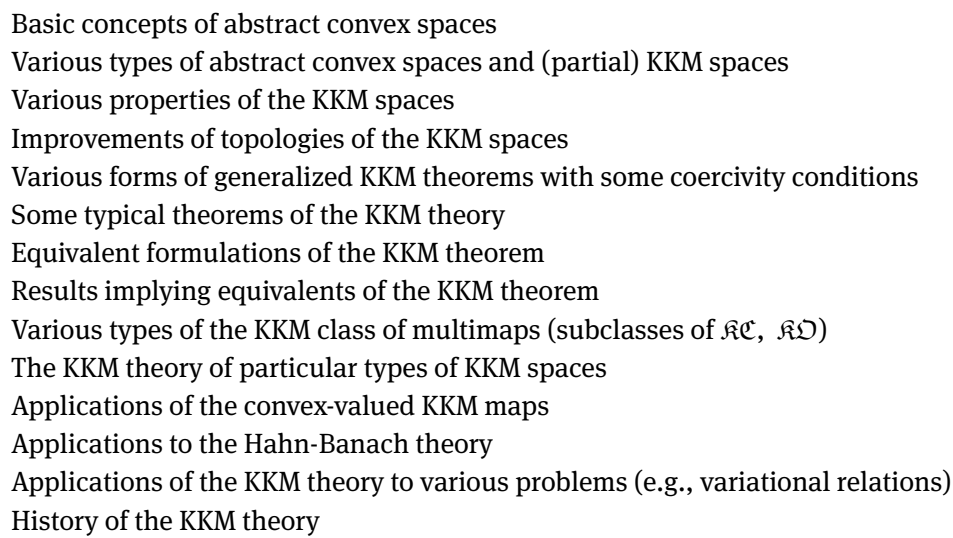

For the references of each of the above subjects, the readers can consult to the publications list of our webpage: http://parksehie.com. Also see Google Scholar and Research Gate. 


\section{Some facts on generalized metric type spaces}

Recall that several authors introduced and studied the following types of generalized metric type spaces:

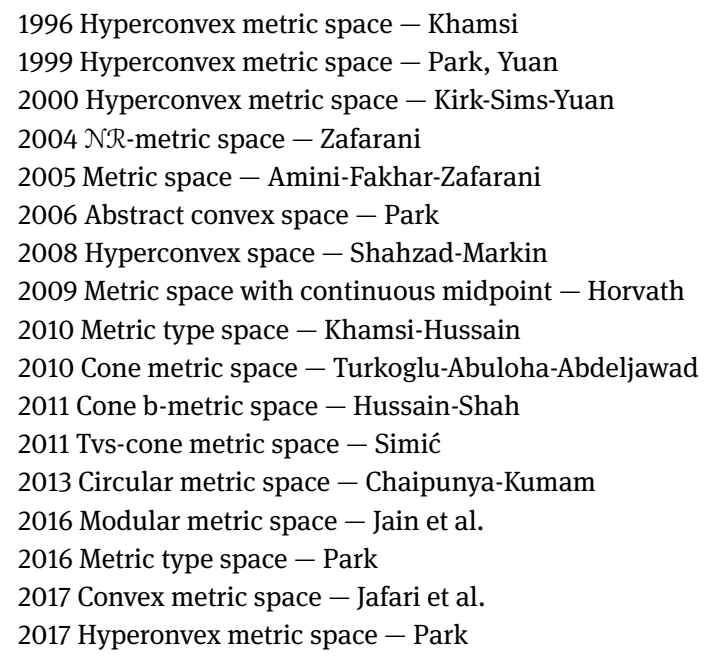

Let $A$ be a nonempty bounded subset of a metric type space $(M, \delta)$. Then we define as follows by adopting Khamsi's way [11]:

(i) $\operatorname{BI}(A)=\operatorname{ad}(A):=\bigcap\{B \subset M \mid B$ is a closed ball in $M$ such that $A \subset B\}$.

(ii) $\mathcal{A}(M):=\{A \subset M \mid A=\operatorname{ad}(A)\}$, i.e., $A \in \mathcal{A}(M)$ iff $A$ is an intersection of closed balls. In this case we will say $A$ is an admissible subset of $M$.

(iii) $A$ is called subadmissible, if for each $N \in\langle A\rangle, \operatorname{ad}(N) \subset A$. Obviously, if $A$ is an admissible subset of $M$, then $A$ must be subadmissible.

For an $x \in M$ and $\varepsilon>0$, let

$$
B(x, \varepsilon):=\{y \in M \mid \delta(x, y) \leq \varepsilon\} \text { and } N(x, \varepsilon):=\{y \in M \mid \delta(x, y)<\varepsilon\} .
$$

It is amazing that, in metric type spaces, when we do not know whether open balls are open and closed balls are closed; see [16].

Here, we need the following extra requirement:

$(\star) \overline{N(x, \varepsilon)} \subset B(x, \varepsilon)$ for all $x \in M$ and $\varepsilon>0$.

This condition holds for any metric space.

We introduce new definitions:

Definition. An abstract convex space $(M, D ; \Gamma)$ is called simply a metric type space if $(M, \delta)$ is a metric type space, $D \subset M$ is a nonempty subset, and $\Gamma:\langle D\rangle \rightarrow \mathcal{A}(M)$ is a map such that $\Gamma_{A}:=\operatorname{BI}(A) \in \mathcal{A}(M)$ for each $A \in\langle D\rangle$. A map $G: D \multimap M$ is a KKM map if $\Gamma_{A} \subset G(A)$ for each $A \in\langle D\rangle$.

A $\Gamma$-convex subset of $(M \supset D ; \Gamma)$ is said to be subadmissible.

Remark. For a metric space $M,(M \supset D ; \Gamma)$ is given in [3], where $\Gamma_{A}:=\operatorname{ad}(A)$. This is a metric type space.

\section{Some articles on generalized metric type spaces}

In this section we introduce some articles which can be improved by following our methods as in the previous sections. Most of these works are chosen on the basis that they can be improved by following our theory. Actually, we introduce abstracts or some part of each paper, and add certain comments like how to improve them. 


\section{Khamsi [11] - JMAA 204 (1996)}

ABSTRACT: In hyperconvex metric spaces, we introduce Knaster-Kuratowski-Mazur- kiewicz mappings. Then we prove an analogue to Ky Fan's fixed point theorem in hyperconvex metric spaces.

Comments: In a metric space $(M, d)$, we denote $B(x, r)$ for the closed ball about $x \in M$ and of radius $r \geq 0$. For a nonempty bounded subset $A \subset M$, set:

$$
\operatorname{co}(A)=\bigcap\{B: B \text { is a closed ball such that } A \subset B\} .
$$

$\mathcal{A}(M)=\{A \subset M: A=\operatorname{co}(A)$, i.e., $A \in \mathcal{A}(M)$ iff $A$ is an intersection of balls. In this case we will say $A$ is an admissible subset of $M$.

With such preparation, Khamsi obtained the following:

Definition 3. Let $H$ be a metric space and $X \subset H$. A multivalued mapping $G: X \rightarrow 2^{H}$ is called a KKM-map if

$$
\operatorname{co}\left(\left\{x_{1}, \ldots, x_{n}\right\}\right) \subset \bigcup_{1 \leq i \leq n} G\left(x_{i}\right)
$$

for any $x_{1}, \ldots, x_{n} \in X$.

Theorem 3. (KKM-Map Principle) Let $H$ be hyperconvex metric space, $X$ an arbitrary subset of $H$, and $G: X \rightarrow$ $2^{H}$ a KKM-map such that each $G(x)$ is finitely closed. Then the family $\{G(x): x \in X\}$ has the finite intersection property.

Note that, according to our current terminology, $(H, X ; \mathrm{co})$ is an abstract convex space by Definition 3 , and it is a KKM space where $H$ has the finitely generated topology. Therefore, it has a large number of properties as shown in Section 3.

In fact, Khamsi showed only a few KKM theoretic properties of $(H, X$; co).

Park [19] - NA 37 (1999)

FROM INTRODUCTION: In this paper, we obtain a Ky Fan type matching theorem for open covers, a coincidence theorem, a Fan-Browder type fixed point theorem, a Brouwer-Schauder-Rothe type fixed point theorem, and other results for hyperconvex spaces. Those are usually obtained for a convex space setting or a generalized convex space setting. Our arguments in this paper are based on the KKM theorem due to Khamsi [11, Theorem 4].

Comments: This paper seems to be a supplement to Khamsi's previous paper in the sense that this adds up few more KKM theoretic properties of hyperconvex metric spaces.

$$
\text { Yuan [37] - JMAA } 235 \text { (1999) }
$$

ABSTRACT: In this note, the characterization for a set-valued mapping with finitely metrically open values being a generalized metric KKM mapping in hyperconvex metric spaces is established. This result could be regarded as a dual form of corresponding results for the Fan-KKM principle in hyperconvex metric spaces obtained recently by M. A. Khamsi [11] and W. A. Kirk, B. Sims, and X. Z. Yuan [14]. Then we show that the finite intersection property of generalized metric KKM mappings with finitely metrically open values indeed is equivalent to the finite intersection property of generalized metric KKM mappings with finitely metrically closed values in hyperconvex spaces. As applications, we first obtain Ky Fan type matching theorems for both closed and open covers in hyperconvex spaces, which, in turn, are used to establish fixed point theorems for set-valued mappings in hyperconvex metric spaces.

Comments: From the summary: "In this paper, a characterization for a set-valued mapping with finitely metrically open values to be a generalized metric KKM mapping in hyperconvex metric spaces is established.”

FROM MR: A hyperconvex metric space is a particular form of a $c$-space (or an H-space) due to C. D. Horvath [Ann. Fac. Sci. Toulouse Math. (6) 2 (1993), no. 2, 253-269; MR1253391 (94i:54043)], and, hence, is a 
generalized convex space (or a G-convex space) due to Park. Therefore, most results in this paper are simple consequences of the corresponding known ones for $c$-spaces or G-convex spaces. Moreover, the author sometimes assumes superfluous restrictions and some of his proofs are unnecessarily lengthy and complicated.

\section{Kirk - Sims - Yuan [14] - NA 39 (2000)}

FROM InTRODUCTION: In this paper, we first establish a characterization of the Knaster-Kuratowski and Mazurkiewicz principle in hyperconvex metric spaces which in turn leads to a characterization theorem for a family of subsets with the finite intersection property in such a setting. As applications we give hyperconvex versions of Fan's celebrated minimax principle and Fanąís best approximation theorem for set-valued mappings. These in turn are applied to obtain formulations of the Browder-Fan fixed point theorem and the Schauder-Tychonoff fixed point theorem in hyperconvex metric spaces for set-valued mappings. Finally, existence theorems for saddle points, intersection theorems and Nash equilibria are also obtained. Our results unify and extend several of the results cited above.

Comments: As we have already seen, Horvath showed in 1993 that hypoconvex metric spaces are his $c$-spaces and hence $G$-convex spaces. However, the KKM theory on $G$-convex spaces was already wellestablished around 1997, and hence, the preceding KKM theoretic studies on hyperconvex metric spaces were out-of-date from the beginning.

$$
\text { Zafarani [28] - Liége 73(2-3) (2004) }
$$

ABSTRACT: In this paper, we give a brief survey of some recent generalization of the Fan-KKM theorem. We introduce a new convex structure on a nonempty set $M$ which contains all different concepts of convexity. Some approximate fixed point theorems will be established for the multivalued mappings with S-KKM property on the $\Phi$ spaces. We also obtain a generalized Fan matching theorem, a generalized Fan-Browder type theorem, and a new version of Sadovskii's fixed point theorem.

Comments: Many results on the KKM theory on various types of spaces are introduced. Zafarani's $\Gamma$ convex spaces are motivated by our G-convex spaces and the same to our original abstract convex spaces in 2006. But he did not establish any theory on his spaces. He adopted a KKM theorem, a very particular form of our Theorem C. Many terms in this paper are obsolete; for example, the KKM class, the S-KKM class, and the generalized S-KKM class belong to our $\mathfrak{K C}$ class in our abstract convex space theory.

Zafarani introduced $N R$-metric spaces, which generalize hyperconvex metric spaces and are G-convex spaces, and hence satisfy all results in Section 3.

Amini - Fakhar - Zafarani [1] - NA 60 (2005)

ABSTRACT: We introduce the class of KKM-type mappings on metric spaces and establish some fixed point theorems for this class. We also obtain a generalized Fan's matching theorem, a generalized Fan-Browder's type theorem, and a new version of Fan's best approximation theorem.

Comments: On the surface, this is a very nice paper. However, the authors adopted inadequate terminology of Chang-Yen. For example, the class of KKM type mapping is $\mathfrak{K C}$ in our works. See the next article.

$$
\text { Park [20] - NAF 11(1) (2006) }
$$

ABSTRACT: We introduce a new concept of abstract convex spaces which are adequate to establish the KKM theory. With this new concept, we can generalize and simplify known results in the theory on convex spaces, H-spaces, G-convex spaces, and others. The KKM type maps are used to obtain coincidence theorems and fixed point theorems. We add a number of examples of abstract convex spaces and generalizations of the KKM principle.

Comments: In this paper, we did not assume any topology for an abstract convex spaces, but we changed our mind later. We also clarified some inadequate usage of terminology; for example, S-KKM class. 


\section{Amini - Fakhar - Zafarani [2] - NA 66 (2007)}

ABSTRACT: We define KKM mappings and S-KKM mappings similarly to in the case of convex spaces for abstract convex spaces. Some approximate fixed point theorems will be established for the multifunction with the S-KKM property on $\Phi$-spaces. We also obtain a new version of Sadovskii's fixed point theorem in topological spaces.

CommenTs: In this paper, we notice the following:

(1) Here abstract convex spaces mean spaces having the routine convexity structure,

(2) Chang - Yen's KKM class [5] should be replaced by the $\mathfrak{K C}$ class.

(3) The S-KKM class of Chang et al. [6] is simply a $\mathfrak{K} \mathfrak{C}$ class; see Park [18].

\section{Shahzad - Markin [33] - JMAA 337 (2008)}

ABSTRACT: In this paper, for a commuting pair consisting of a point-valued nonexpansive self-mapping $t$ and a set-valued nonexpansive self-mapping $T$ of a hyperconvex metric space (or a CAT(0) space) $X$, we look for a solution to the problem of existence of $z \in E \subset X$ such that $d(z, y)=d(y, E)$ and $z=t(z) \in T(z)$.

ComMENTS: Hyperconvex metric spaces and complete CAT(0) spaces are Horvath spaces and hence KKM spaces. Therefore, they have many properties as shown in Section 3.

\section{Horvath [7] - Romanian 1(2) (2009)}

ABSTRACT: A metric space $(X, d)$ is a continuous midpoint space if there is a continuous map $\mu: X \times$ $X \rightarrow X$ such that, for all $(a, b) \in X \times X, d(a, \mu(a, b))=(1 / 2) d(a, b)=d(b, \mu(a, b))$. A closed subset $\mathrm{C}$ of a complete continuous midpoint space is convex if $\forall(a, b) \in C \times C, \mu(a, b) \in C$. Under suitable, but natural, assumptions continuous midpoint spaces are absolute retracts; Browder, Michael or Cellina like continuous selection theorems hold; bounded closed convex sets have the fixed point property for nonexpansive maps. Hyperconvex metric spaces, Cartan - Hadamard manifolds and more generally Hadamard spaces or metric spaces with non positive curvature in the sense of Busemann are continuous midpoint spaces.

Turkoglu et al. [36] - NA 72 (2010)

ABSTRACT: In this paper, we define KKM mappings in cone metric spaces and define NR-cone metric spaces to obtain some fixed point theorems and hence generalize the results obtained by A. Amini, M. Fakhar, J. Zafarani [1].

Comments: For a cone metric space $(M, d)$, adopting Khamsi's terminology, Turkoglu et al. [36] take the Chang - Yen KKM multimap class and the compactly generated topology. These should be modernized.

For a cone metric space $(M, d)$ and a nonempty subadmissible subset $X$ of $M$, they obtained:

Theorem 13. If $F \in \mathfrak{K C}(X, X)$ and $c l F(X)$ is totally bounded, then $F$ has the approximate fixed point property. Theorem 14. Suppose that $F \in \mathfrak{K} \mathfrak{C}(X, X)$ is closed and compact, then $F$ has a fixed point.

Lemma 16. If $Y$ is a topological space, $F \in \mathfrak{K C}(X, Y)$, and $f: Y \rightarrow X$ is continuous, then $f F \in \mathfrak{K C}(X, X)$.

Corollary 17. Suppose that the identity mapping $1_{X} \in \mathfrak{K C}(X, X)$, then any continuous mapping $f: X \rightarrow X$ such that $\operatorname{cl} f(X)$ is compact, has a fixed point.

Some of the above results extend corresponding ones of Amini et al. [1] for metric spaces. Moreover, Lemma 16 has already a far-reaching extended form in Park [21], see Theorem D in Section 2.

Khamsi - Hussain [12] - NA 73 (2010)

ABSTRACT: In this work we discuss some recent results about KKM mappings in cone metric spaces. We also discuss the fixed point existence results of multivalued mappings defined on such metric spaces. In particular we show that most of the new results are merely copies of the classical ones and do not necessitate the underlying Banach space nor the associated cone. 
FROM INTRODUCTION: It is worth mentioning the pioneering work of Quilliot (in 1983) who introduced the concept of generalized metric spaces. His approach was very successful and is used by many (see the references in Jawhari et al. in 1986). It is our belief that cone metric spaces are a special case of generalized metric spaces. In this work, we introduce a metric type structure in cone metric spaces and show that classical proofs related to KKM mappings proved in [35] do apply almost identically in these metric type spaces. This approach suggests that any extension of known fixed point results to cone metric spaces is redundant. Moreover the underlying Banach space and the associated cone subset are not necessary.

Hussain - Shah [8] - CMA 62 (2011)

ABSTRACT: In this paper we establish some topological properties of the cone b-metric spaces and then improve some recent results about KKM mappings in the setting of a cone $b$-metric space. We also prove some fixed point existence results for multivalued mappings defined on such spaces.

FROM INTRODUCTION: In this work, with the structure of a cone b-metric space, we shall establish some topological properties of the cone b-metric spaces. We also prove and extend some results of Khamsi and Hussain [12] and illustrate our work in this setting with examples.

CommEnTs: The authors also adopted the obsolete Chang - Yen KKM class.

For a cone b-metric space $(M, D)$ and a nonempty subadmissible subset $X$ of $M$, a few results of the previous work of Turkoglu et al. [36] are extended. For example,

Theorem 4.4. Let $X$ be a nonempty subadmissible subset of $M$. Suppose that $X$ is a partial KKM space, then any compact continuous map $f: X \rightarrow X$ has a fixed point.

Simić [35] - FPTA 62 (2011)

ABSTRACT: Recently, Ayse Sonmez [On paracompactness in cone metric spaces, Appl. Math. Lett. 23 (2010) 494-497] proved that a cone metric space is paracompact when the underlying cone is normal. Also, very recently, Kieu Phuong Chi and Tran Van An [Dugundji's theorem for cone metric spaces, Appl. Math. Lett. (2010) doi:10.1016/j.aml.2010.10.034] proved Dugundji's extension theorem for the normal cone metric space. The aim of this paper is to prove this in the frame of the tvs-cone spaces in which the cone does not need to be normal. Examples are given to illustrate the results.

CommEnTs: The author showed that any cone normed space $(X, \||\cdot| \mid))$ is a partial KKM space and, hence, satisfies a large number of the KKM theoretic results in Section 3.

Chaipunya - Kumam [3] - JIA 2013:343 (2013)

ABSTRACT: The main purpose of this paper is to study some topological nature of circular metric spaces and deduce some fixed point theorems for maps satisfying the KKM property. We also investigate the solvability of a variant of a quasi-equilibrium problem as an application.

CommEnTs: In this paper, let $M$ be a circular metric space and $X$ be a subadmissible subset of $M$. A multimap $G: X \multimap M$ is said to be a KKM map if for each $A \in X$ we have $\operatorname{ad}(A) \subset G(A)$.

Note that $(M, X ; a d)$ is an abstract convex space.

In this paper, as in the many metric type spaces, inadequate $K K M$ class instead of $\mathfrak{K C}$ is used. Certain results can be improved by adopting current language in the KKM theory.

Park [27] - JNAS-ROK 54(2) (2015)

ABSTRACT: For a long period, the study of hyperconvex metric spaces introduced by Aronszajn and Panitchpackdi in 1956 was concentrated to the fixed point property of nonexpansive maps. However, since Khamsi in 1996 found a KKM theorem for such spaces, there have appeared a large number of works on them related to the KKM theory. In the present review, we follow the various stages of developments of the KKM theory of hyperconvex metric spaces. In fact, we introduce abstracts of articles on such theory and give comments or 
generalizations of the results there if necessary. We show that many results in those articles are consequences of (partial) KKM space theory developed by ourselves from 2006.

Comments: While preparing this paper, we noticed some papers on useless studies of the KKM theory and they were criticized in several papers; for example [26].

Jain et al. [10] - MDPI (2016)

ABSTRACT: The purpose of this paper is to present a new approach to study the existence of fixed points for multivalued F-contraction in the setting of modular metric spaces. In establishing this connection, we introduce the notion of multivalued F-contraction and prove corresponding fixed point theorems in complete modular metric space with some specific assumption on the modular. Then we apply our results to establish the existence of solutions for a certain type of non-linear integral equations.

CommEnTs: No KKM theoretic result was given in this paper.

Park [28] - LNA 2(1) (2016)

ABSTRACT: Recent results in the KKM theory of abstract convex spaces and the related multimap classes $\mathfrak{K C}$ and $\mathfrak{K O}$ are applied to deduce generalizations of results on KKM maps in metric type spaces of Khamsi and Hussain [12].

FROM INTRODUCTION: Recently, Khamsi and Hussain [12] introduced a metric type structure in cone metric spaces and showed that classical proofs related to KKM maps proved in [36] do carry almost identically in these metric type spaces. This approach suggests that any extension of known fixed point results to cone metric spaces is redundant.

We found that there are some similar results in $[12,13,29]$. Our principal aim in this article is to obtain generalized results unifying the corresponding ones in [12] and [22].

Shabanian - Vaezpour [34] - B.Malays 39 (2016)

ABSTRACT: In this paper, we present a modular version of KKM and generalized KKM mappings and then we establish a characterization of generalized KKM mappings in modular spaces. Also we prove an analogue to KKM principle in modular spaces. Moreover, as an application, we give some sufficient conditions which guarantee existence of solutions of minimax problems in which we get Fan's minimax inequality in modular spaces.

Comments: Generalized KKM maps defined by Chang - Zhang [4] in 1991 were extended by several authors. Finally, Lee [15] in 2016 showed that they are simply KKM maps in abstract convex spaces.

In 2016, Shabanian - Vaezpour [34] characterized generalized KKM map in modular spaces and obtained

Corollary 3.4. Let $\rho$ be a modular on $Y, X$ be a nonempty set of $Y_{\rho}$, and $G: X \multimap Y_{\rho}$ be a closed-valued map. If $G$ is $K K M$, then the family $\{G(x): x \in X\}$ has the finite intersection property.

This means that $\left(Y_{\rho}, X\right.$; co) is a partial KKM space and, hence, satisfies a large number of KKM theoretic facts in Section 3.

Jafari et al. [9] - Optimization 66(3) (2017)

ABSTRACT: This paper deals with equilibrium problems in the setting of metric spaces with a continuous convex structure. We extend Fan's 1984 KKM theorem to convex metric spaces in order to employ some weak coercivity conditions to establish existence results for suitable local Minty equilibrium problems, where the involved bifunctions are $\varphi$-quasimonotone. By an approach which is based on the concept of the strong $\varphi$ sign property for bifunctions, we obtain existence results for equilibrium problems which generalize some results in the literature. 
Comments: Fan's 1984 KKM theorem can be extended to Theorem C in Section 2 and a convex metric space is a partial KKM space. Therefore, a convex metric space satisfies many properties in Section 3.

$$
\text { Park [30] - NAF 22(2) }
$$

ABSTRACT: In 1996, Khamsi established the KKM theorem for hyperconvex metric spaces and applied it to obtain a Schauder type fixed point theorem. This line of study has been followed by a large number of authors. In this article, we show that the KKM theorem, best approximation theorem, and the Schauder type fixed point theorem for hyperconvex metric spaces due to Khamsi can be extended to partial KKM metric spaces.

$$
\text { Park [31] - RNA 2(2) (2019) }
$$

ABSTRACT: Since Khamsi found a KKM theorem for hyperconvex metric spaces in 1996, there have appeared a large number of works on them related to the KKM theory. In our previous review [42], we followed the various stages of developments of the KKM theory of hyperconvex metric spaces. In fact, we introduced abstracts of articles on such theory and gave comments or generalizations of the results there if necessary. We noted that many results in those articles are consequences of (partial) KKM space theory developed by ourselves from 2006. The present survey is a continuation of [42] and aims to collect further generalizations of hyperconvex metric spaces related to the KKM theory.

Mitrović et al. [16] - MPDA

ABSTRACT: In this paper we obtain a best approximations theorem for set-valued mappings in G-convex spaces. As applications, we derive results on the best approximations in hyperconvex and normed spaces. The obtain results generalize many known results in the literature.

Comments: G-convex spaces are obsolete now.

\section{Conclusion}

The study of generalized metric type spaces seems to be quite active. From Khamsi's pioneering work, most of the results are closely related to the KKM theory initiated by the present author. However, we note that the following will be helpful to peoples working this field:

1. Most of works on generalized metric type spaces do not reflect current states of the KKM theory.

2. Most of authors does not know the mapping classes $\mathfrak{K C}$ and $\mathfrak{K O}$, and adopted the obsolete class $K K M$ due to Chang and Yen.

3. Most of authors does not know the concepts of KKM spaces or partial KKM spaces having a large number of properties as shown in Section 3.

4. Generalized metric type spaces should have some special characters other than the KKM theoretic results in Section 3.

5. Most of results on generalized metric type spaces seem to be similar ones imitating some previous ones.

\section{References}

[1] Amini, A., Fakhar, M. and Zafarani, J. KKM mappings in metric spaces, Nonlinear Anal. 60 (2005) 1045-1052.

[2] Amini, A., Fakhar, M. and Zafarani, J. Fixed point theorems for the class S-KKM mappings in abstract convex spaces, Nonlinear Anal. 66 (2007) 14-21. 
[3] Chaipunya, P. and Kumam, P. Topological aspects of circular metric spaces and some observations on the KKM property towards quasi-equilibrium problems, J. Ineq. Appl. 2013, 2013:343

[4] Chang, S.-S. and Zhang, Y. Generalized KKM theorem and variational inequalities, J. Math. Anal. Appl. 159 (1991) $208-223$.

[5] Chang, T.H. and Yen, C.L. KKM property and fixed point theorems, J. Math. Anal. Appl. 203 (1996) 224-235.

[6] Chang, T.H., Huang, Y.Y., Teng, J.C., and Kuo, K.W. On the S-KKK property and related topics, J. Math. Anal. Appl. 229 (1999) 212-227.

[7] Horvath, C. D. A note on metric spaces with continuous midpoints, Annal. Acad. Rumanian Scientists, Ser. Math. Appl. 1(2) (2009) 252-288.

[8] Hussain, N. and Shah, M. H. KKM mappings in cone b-metric spaces, Comp. Math. Appl. 62 (2011) 1677-1684.

[9] Jafari, S., Farajzadeh, A. P., Moradi, S. and Khanh, P. Q. Existence results for $\varphi$-quasimonotone equilibrium problems in convex metric spaces, Optimization 66(3) (2017) 293-310. http://dx.doi.org/10.1080/02331934.2016.1274989

[10] Jain, D., Padcharoen, A., Kumam, P. and Gopal, D. A new approach to study fixed point of multivalued mappings in modular metric spaces and applications, MDPI 2016.

[11] Khamsi, M. A. KKM and Ky Fan theorems in hyperconvex metric spaces, J. Math. Anal. Appl. 204 (1996) 298-306.

[12] Khamsi, M. A. and Hussain, N. KKM mappings in metric type spaces, Nonlinear Anal. 73 (2010) 3123-3129.

[13] Khamsi, M. A., Latif, A. and Al-Sulami, H. KKM and Ky Fan theorems in modular function spaces, Fixed Point Theory Appl. 2011, 2011:57

[14] Kirk, W. A., Sims, D. and Yuan, G. X.-Z. The Knaster-Kuratowski and Mazurkiewicz theory in hyperconvex metric spaces and some of its applications, Nonlinear Anal. 39 (2000) 611-627.

[15] Lee, W. A remark on generalized KKM maps on KKM spaces, Nonlinear Analysis Forum 21(1) (2016) 1-5.

[16] Mitrović, Z. D., Hussain, A., de la Sen, M., and Radenovi, S. On best approximations for set-valued mappings in G-convex spaces, MPDA, 2020.

[17] Park, S. Some coincidence theorems on acyclic multifunctions and applications to KKM theory, Fixed Point Theory and Applications (K.-K. Tan, ed.), pp.248-277, World Scientific Publ., River Edge, NJ, 1992.

[18] Park, S. Ninety years of the Brouwer fixed point theorem, Vietnam J. Math. 27 (1999), 187-222.

[19] Park, S. Fixed point theorems in hyperconvex metric spaces, Nonlinear Anal. 37 (1999), 467-472.

[20] Park, S. On generalizations of the KKM principle on abstract convex spaces, Nonlinear Anal. Forum 11(1) (2006) 67-77.

[21] Park, S. Examples of $\mathfrak{K C}$-maps and $\mathfrak{K O}$-maps on abstract convex spaces, Soochow J. Math. 33(3) (2007) 477-486.

[22] Park, S. Comments on the KKM theory on hyperconvex metric spaces, Tamkang J. Math. 41(1) (2010) 1-14.

[23] Park, S. The KKM principle in abstract convex spaces: Equivalent formulations and applications, Nonlinear Anal. 73 (2010) 1028-1042.

[24] Park, S. New generalizations of basic theorems in the KKM theory, Nonlinear Anal. 74 (2011) 3000-3010.

[25] Park, S. A genesis of general KKM theorems for abstract convex spaces: Revisited, J. Nonlinear Anal. Optim. 4(2) (2013) 127132.

[26] Park, S. Comments on the FWC-spaces of H. Lu and J. Zhang, Nonlinear Anal. Forum 18 (2013) 33-38.

[27] Park, S. Evolution of the KKM Theory of hyperconvex spaces, J. Nat. Acad. Sci., ROK, Nat. Sci. Ser. 54(2) (2015) 1-28.

[28] Park, S. Comments on the KKM theory of metric type spaces, Linear and Nonlinear Anal. 2(1) (2016) 39-45. MR3574137.

[29] Park, S. A history of the KKM Theory, J. Nat. Acad. Sci., ROK, Nat. Sci. Ser. 56(2) (2017) 1-51.

[30] Park, S. Generalizations of Khamsi's KKM and fixed point teorems on hyperconvex metric spaces, Nonlinear Anal. Forum 22(2) (2017) 7-15.

[31] Park, S. Generalizations of hyperconvex metric spaces, Results in Nonlinear Analysis 2(2) (2019) 71-82.

[32] Park, S. Improving some KKM theoretic results, Results in Nonlinear Analysis 3(2) (2020) 68-77.

[33] Shahzad, N. and Markin, J. Invariant approximations for commuting mappings in CAT(O) and hyperconvex spaces, J. Math. Anal. Appl. 337 (2008) 1457-1464.

[34] Shabanian, S. and Vaezpour, S. M. The KKM theorem in modular spaces and applications to minimax inequalities, Bull. Malays. Math. Sci. Soc. 39 (2016) 921-931. DOI 10.1007/s40840-015-0192-3

[35] Simić, S. A note on Stone's, Baire's, Ky Fan's and Dugundji’s theorem in tvs-cone metric spaces, Appl. Math. Lett. 24 (2011) 999-1022.

[36] Turkoglu, D., Abuloha, M. and Abdeljawad, T. KKM mappings in cone metric spaces and some fixed point theorems, Nonlinear Anal. 72 (2010) 348-353.

[37] Yuan, G. X.-Z. The characterization of generalized metric KKM mappings with open values in hyperconvex metric spaces and some applications, J. Math. Anal. Appl. 235(1) (1999) 315-325.

[38] Zafarani, J. KKM property in topological spaces, Bull. Soc. Roy. Sci. Liége 73(2-3) (2004) 171-185. 